\title{
The $4 \mathrm{q} 25,1 \mathrm{q} 21$, and $16 \mathrm{q} 22$ polymorphisms and recurrence of atrial fibrillation after pulmonary vein isolation
}

Marek Kiliszek ${ }^{1}$, Edward Kozluk ${ }^{1}$, Maria Franaszczyk², Piotr Lodzinski ${ }^{1}$, Agnieszka Piatkowska ${ }^{1}$, Rafal Ploski ${ }^{3}$, Grzegorz Opolski ${ }^{1}$

\section{${ }^{1}$ First Chair and Department of Cardiology, Medical University of Warsaw, Warsaw, Poland \\ 2Laboratory of Molecular Biology, Institute of Cardiology, Warsaw, Poland \\ ${ }^{3}$ Department of Medical Genetics, Medical University of Warsaw, Warsaw, Poland}

Submitted: 7 February 2014

Accepted: 20 April 2014

Arch Med Sci 2016; 12, 1: 38-44

DOI: $10.5114 /$ aoms.2015.48284

Copyright ( 2016 Termedia \& Banach

\section{Abstract}

Introduction: The efficacy of pulmonary vein isolation (PVI) in atrial fibrillation (AF) is well documented. Several single nucleotide polymorphisms (SNPs) are associated with AF, mainly in the $4 q 25$ locus, but also in $16 q 22$ and $1 \mathrm{q} 21$. The aim of our study was to test the association between those SNPs and short- and long-term results of PVI.

Material and methods: Patients with AF who underwent PVI between 2006 and 2009 were included in the study. Pulmonary vein isolation was performed using a 4-mm non-irrigated ablation catheter, circular mapping catheter, and the LocaLisa system. All patients were genotyped for the $4 q 25$, 16q22, and 1q21 SNPs.

Results: Two-hundred and thirty-eight patients were included. The median follow-up was 45 months. Six-month efficacy was $59.7 \%$. None of the polymorphisms was linked with the risk of AF recurrence after 6 months in univariate analysis. In multivariate analysis rs2200733 in the recessive model was linked significantly with AF recurrence (odds ratio 1.87, $p=0.008$ ). None of the polymorphisms predicted AF recurrence in long-term follow-up. Conclusions: There is a trend in the relationship between TT genotype of the rs2200733 polymorphism and increased rate of AF recurrence after PVI in short-term (6 months) follow-up. None of the tested SNPs 4q25, 16q22, and $1 \mathrm{q} 21$ correlated with the results of a single AF ablation in long-term follow-up.

Key words: genetic polymorphism, pulmonary vein isolation, atrial fibrillation, catheter ablation.

\section{Introduction}

Catheter ablation is a standard therapy in many cases of atrial fibrillation (AF) [1], with well-documented efficacy (mainly with pulmonary vein isolation - PVI) [2]. The molecular background of AF is not yet clear, even in diseases with a cause-effect relationship with AF, such as hyperthyroidism [3]. Several single-nucleotide polymorphisms (SNPs) have been however associated with $\mathrm{AF}$, with the major loci located on chromosome $4 \mathrm{q} 25$ (SNPs: rs2200733, rs10033464, rs17570669, rs3853445, rs6838973) $[4,5]$ and less strongly associated loci on 16q22 (rs7193343) and 1q21

\author{
Corresponding author: \\ Marek Kiliszek \\ First Chair and Department \\ of Cardiology \\ Medical University of Warsaw \\ 1 a Banacha St \\ 02-097 Warsaw, Poland \\ Phone: +48 225992958 \\ Fax: +48 225991957 \\ Department of Cardiology \\ and Internal Diseases \\ Military Institute of Medicine \\ 128 Szaserów St \\ 04-141 Warsaw, Poland \\ Phone: +48 216817909 \\ E-mail: kiliszek@mp.pl
}


(rs13376333) [6, 7]. The association with the $4 q 25$ SNPs has a plausible molecular explanation: the closest gene, PITX2, encodes a transcription factor that influences heart development, especially the pulmonary veins $[4,8]$. All mentioned associations have been confirmed in a study of patients from our centre - we have shown that patients with AF have significantly higher frequency of the $4 q 25$ (especially rs2200733), 16q21 and 1q21 variants than the control group [9].

A previous study showed a link between the two 4q25 chromosome SNPs most strongly associated with $\mathrm{AF}$ and the short-term results of catheter ablation [10]. Since prediction of AF recurrence is an important issue, especially in longterm observation [11], the reported results were intriguing.

The aim of our study was to revisit the reported association between the 6-month outcome of ablation and the two most potent $4 q 25$ SNPs (rs2200733 and rs10033464) in another population [10]. We also extended the analysis to include the remaining SNPs associated with AF (rs17570669, rs3853445, rs6838973, rs7193343 and rs13376333), as well as a longer follow-up period after catheter ablation.

\section{Material and methods}

\section{Study population}

A prospective cohort study was performed, with genetic analysis available at the end of the observation period. Consecutive patients with AF (paroxysmal or persistent) who underwent pulmonary vein isolation in the years 2006 to 2009 were included in the study. Inclusion criteria were as follows: symptomatic AF without reversible cause, unsuccessful treatment with at least one antiarrhythmic drug (group Ic or III), and age below 70 years. Active hyperthyroidism, significant mitral valve disease, left atrial dimension over $5.5 \mathrm{~cm}$, or severe disease with life expectancy below 1 year were the exclusion criteria.

The study was approved by the Bioethics Committee of the Medical University of Warsaw, and all patients gave written informed consent.

\section{Ablation strategy}

Ablations were performed at the Medical University of Warsaw in the years 2006 to 2009.

One quadripolar catheter was placed in the coronary sinus and one in the right ventricle. The left atrium was accessed through one transseptal puncture (or patent foramen ovale, if present), and a 10-pole circumferential 15-25 mm-Lasso (Biosense Webster, Diamond Bar, Ca, USA) or Optima (St Jude Medical, Minnetonka, MN, USA) and 4-mm non-irrigated tip ablation (Marinr, Medtron- ic, Minneapolis, MN, USA) catheters were used for mapping and radiofrequency ablation. After transseptal puncture, the patients were heparinized throughout left atrial access. The placement of the catheters in the heart was based on fluoroscopy and the electroanatomical LocaLisa system. Radiofrequency energy was delivered in the temperature control mode, with a temperature limit of $55^{\circ} \mathrm{C}$ and a power limit of $35 \mathrm{~W}$. All electrograms were displayed on an electrophysiological recording system. The endpoint of the procedure was to isolate pulmonary vein potentials in all pulmonary veins (in paroxysmal and persistent AF patients). No additional lines or applications in the left atrium were performed. In most of the cases, if the patient was on atrial fibrillation, cardioversion was performed to verify isolation during sinus rhythm. After pulmonary vein isolation, in patients with paroxysmal AF on sinus rhythm isolation of vena cava superior (VCS) potentials was performed using the same mapping and ablation catheters.

\section{Follow-up}

According to current guidelines, a recurrence of AF was defined as any atrial tachycardia lasting more than $30 \mathrm{~s}$ with a 3-month blanking period applied [1]. In paroxysmal AF patients, antiarrhythmic drugs (AAD) were discontinued immediately after catheter ablation. In the case of persistent or long-term persistent AF in patients without recurrences of $A F, A A D$ were discontinued 1 year after ablation. Treatment with vitamin $\mathrm{K}$ antagonists (VKA) was continued for 3 months (patients with a CHADS score of 0 ), 1 year (CHADS 1 ), or indefinitely (CHADS 2). During the first year after ablation, 6 days of ECG Holter monitoring was recommended (1 day every 2 months; median number of days of Holter monitoring 1 year after ablation in patients without recurrences was 3; IQR 1-6). Further monitoring was performed at the discretion of the outpatient cardiologist. Final follow-up was based on patient visits, telephone contact, and analysis of Holter monitoring and/or other patient documentation. In the case of recurrence, the decision whether to repeat the procedure was based on clinical symptoms and patient preferences. The method of the repeat procedure was individually decided by the operator.

\section{Genotyping}

Genomic DNAwasisolated from peripheral blood by salting out. Genotyping of SNPs rs2200733, rs10033464, rs17570669, rs3853445, rs6838973, rs7193343, and rs13376333 was performed using TaqMan Assays (C_16158671_10, Custom TaqMan SNP Genotyping Assay, C_33254659_10, 
C_1176985_10,C_29128132_20,C_29343982_10 and C_2745708_10, respectively) (Applied Biosystems, Foster City, Cal, USA). Assays were performed according to the manufacturer's instructions using a 7500 Real-Time PCR System (Applied Biosystems).

\section{Statistical analysis}

Continuous variables are presented as mean \pm standard deviation (SD) or median $\left(1^{\text {st }}-3^{\text {rd }}\right.$ quartile). Categorical variables are presented as frequencies. The $\chi^{2}$ or Fisher's exact tests were used to test deviations of the genotype distribution from Hardy-Weinberg equilibrium and to compare allele and genotype frequencies between groups. Effects of genotypes were analyzed under dominant (wild type vs. patients with polymorphic allele), additive (wild type vs. heterozygotes with polymorphic allele vs. polymorphic homozygotes), and recessive (polymorphic homozygotes vs. polymorphic heterozygotes and wild type) models using the online tool available at http://www.ekstroem.com/assotest/assotest.html [12]. We used Bonferroni correction for multiple genetic testing; a $p$ value $0.05 / 19=0.0026$ was considered statistically significant in univariate analysis. The test for the effect of the T allele at either rs2200733 or rs10033464 on AF recurrence was hypothesis driven [10] and the $p$ value was not corrected for number of comparisons. Multivariate logistic regression was performed to assess independent significance of all parameters with a $p$ value $<0.05$ in univariate analysis. In long-term analysis Cox regression was used to test the relationship between clinical factors, genetic polymorphisms and AF recurrence after ablation. Our study had the power of over $90 \%(\alpha=0.05)$ to detect an effect of the T allele at either rs2200733 or rs10033464 on AF recurrence, as reported by Husser et al. [10].

\section{Results}

Two-hundred and thirty-eight patients with at least 1 year of follow-up were included in the study group. Characteristics of the study group are shown in Table I. Median follow-up was 45 months (interquartile range 32-57) and the total number of procedures was 349 (1.47 per patient).

\section{Six-month follow-up}

After 6 months 142 patients remained without AF (59.7\%). Clinical factors predicting recurrence in univariate analysis are shown in Table I. In uni-

Table I. Clinical characteristics of the studied group and subgroups defined by AF recurrence within 6 months

\begin{tabular}{|c|c|c|c|c|}
\hline Characteristic & $\begin{array}{c}\text { All subjects } \\
n=238\end{array}$ & $\begin{array}{c}\mathrm{R}^{\ddagger}(+) \\
n=96\end{array}$ & $\begin{array}{c}\mathrm{R}^{\ddagger}(-) \\
n=142\end{array}$ & $P$-value* \\
\hline Female gender & $79(33.2)$ & $37(38.5)$ & $42(29.6)$ & 0.16 \\
\hline Age, median (IQR) [years] & $55(47-61)$ & $56(49-61)$ & $55(46-61)$ & 0.22 \\
\hline Body mass index $\left[\mathrm{kg} / \mathrm{m}^{2}\right]$ & $27.8(25.5-31.1)$ & $27.6(24.5-31.5)$ & $28.3(25.8-31.1)$ & 0.58 \\
\hline Procedure time [min] & $130(105-155)$ & $135(110-160)$ & $120(105-152.5)$ & 0.045 \\
\hline Fluoroscopy time $[\mathrm{s}]$ & $973(681-1531)$ & $1013.5(752-1569)$ & $963(655-1460)$ & 0.26 \\
\hline RF application time [s] & $2655(2019-3360)$ & $2708(2105-3540)$ & $2645(1924-3270)$ & 0.22 \\
\hline Isolation of vena cava superior ${ }^{\dagger}$ & $148(62.2)$ & $51(53.1)$ & $97(68.3)$ & 0.021 \\
\hline AF duration & $5(3-8)$ & $5(3-9)$ & $5(3-7)$ & 0.61 \\
\hline Persistent AF & $36(15.1)$ & $13(13.5)$ & $23(16.2)$ & 0.71 \\
\hline Lone AF & $75(31.5)$ & $29(30.2)$ & $46(32.4)$ & 0.78 \\
\hline AF within $48 \mathrm{~h}$ after ablation & $68(28.6)$ & $35(36.5)$ & $33(23.2)$ & 0.029 \\
\hline Coronary artery disease & $24(10.1)$ & $13(13.5)$ & $11(7.7)$ & 0.19 \\
\hline Hypertension & $139(58.4)$ & $57(59.4)$ & $82(57.7)$ & 0.89 \\
\hline Diabetes & $18(7.6)$ & $11(11.4)$ & $7(4.9)$ & 0.080 \\
\hline Heart failure & $3(1.3)$ & $1(1.0)$ & $2(1.4)$ & 0.57 \\
\hline Left atrium dimension & $4.13(0.50)$ & $4.16(0.45)$ & $4.13(0.49)$ & 0.73 \\
\hline Hyperlipidemia & $51(21.4)$ & $22(22.9)$ & $29(20.4)$ & 0.74 \\
\hline
\end{tabular}

Results were presented as $n$ (\%). ${ }^{*} t$ test for continuous variables, $\chi^{2}$ for dichotomous variables; comparison between the group with $A F$ recurrence within 6 months vs. those without; $p$ values $<0.05$ are boldfaced; ${ }^{\dagger}$ during the same procedure, with pulmonary vein isolation; ${ }^{\ddagger}$ recurrence. 
variate genetic analysis none of the parameters was significantly associated with AF recurrence. Two 4q25 polymorphisms showed an association $\left(p_{\text {not corrected }}<0.05\right)$ and were included in the multivariate analysis (Table II). We did not find any association between 6-month AF recurrence and presence of the $\mathrm{T}$ allele at either rs2200733 or rs10033464 (Table III). In multivariate analysis two factors significantly predicted AF recurrence: VCS isolation and rs2200733 in the recessive model (Table IV).

\section{Long-term follow-up}

Seventy-seven (32.3\%) patients were continuously free of arrhythmia in the long term after a single procedure. None of the polymorphisms significantly predicted the recurrence of AF after a single procedure (data not shown).

\section{Discussion}

The main finding is lack of replication of previously published results [10]. Our results suggest that there might be in the relationship between rs2200733 in the recessive model (non-significant result in univariate analysis after Bonferroni correction, significant result in multivariate analysis) and the results of AF ablation in short-term (6 months) observation, but not in the long term (median of 45 months). Based on the frequency of AF recurrence in a previous publication [10], our study had power exceeding $90 \%$ to detect relationships between rs2200733, rs10033464 and recurrence of AF.

The major allele frequency in our group was comparable to that reported in the literature in AF groups, except rs2200733 [4-7]. This polymorphism was more frequent than reported so far (0.33 in our population vs. 0.21 to 0.27 as previously reported $[5,10])$. We speculated earlier that patients qualified to catheter ablation are usually drug resistant and highly symptomatic [9] and this could have caused such a difference. In this study we did not have a control group, but our previous study showed that the major allele frequency of all tested polymorphisms in controls without AF is comparable with reports published so far on populations of European ancestry [4, 5, 9].

In patients with the TT genotype of the $4 q 25$ rs2200733 polymorphism there was an increased rate of $A F$ recurrence after catheter ablation in short-term but not in long-term follow-up. The other 4q25 polymorphisms (rs10033464, rs3853445, rs17570669 and rs6838973) and other loci with weaker association with AF (1q21 and 16q22) showed no correlation with the results of PVI. Thus, recurrences might be influenced by genetic factors, while late recurrences (occurring later than 1 year after ablation) are probably not.

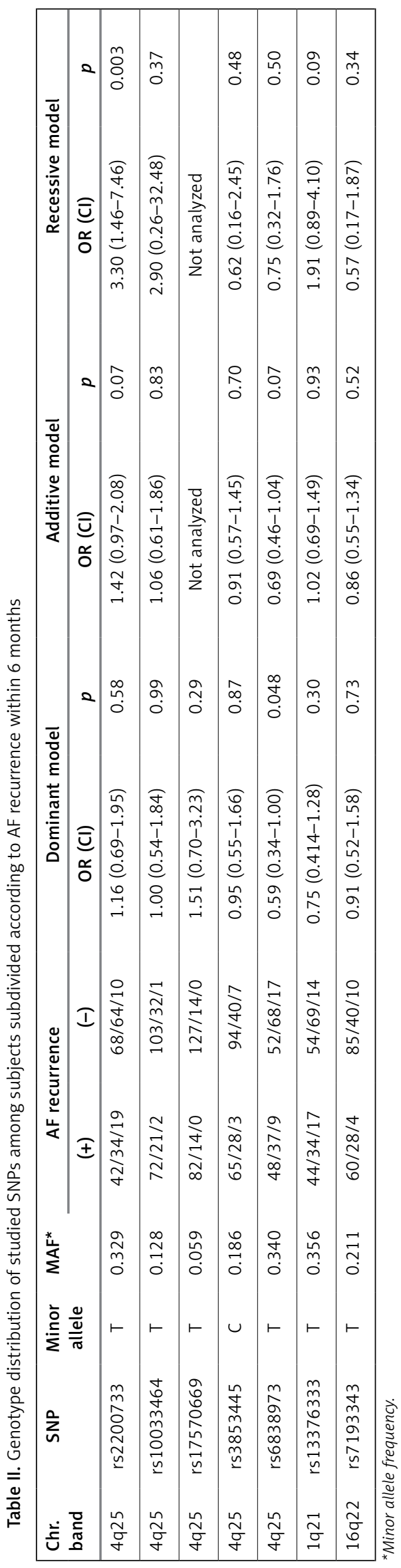


Table III. Prevalence of patients carrying the AF risk allele (T) at either rs2200733 or rs10033464 after stratification according to AF recurrence

\begin{tabular}{|c|c|c|}
\hline AF recurrence & $\begin{array}{c}\text { rs2200733 T } \\
\text { or rs10033464 T } \\
n(\%)\end{array}$ & OR (CI), $p$-value \\
\hline \multicolumn{3}{|l|}{ Within 48 h: } \\
\hline Yes & $45(67.1)$ & \multirow{2}{*}{$\begin{array}{c}0.78(0.42-1.43) \\
p=0.51\end{array}$} \\
\hline No & 119 (72.6) & \\
\hline \multicolumn{3}{|c|}{ Within 6 months: } \\
\hline Yes & $68(71.6)$ & \multirow{2}{*}{$\begin{array}{c}0.95(0.53-1.70) \\
p=0.98\end{array}$} \\
\hline No & $96(70.6)$ & \\
\hline
\end{tabular}

Our results underscore the differences between those recurrences. Although according to current guidelines, pulmonary vein reconduction is very common in patients with recurrences and late recurrences, numerous investigators suggest that very late recurrences of AF may reflect alterations in the substrate of AF and impact of diseases such as hypertension, sleep apnea or diabetes on the progress of AF [13].

Our results are less optimistic than the results in the original publication on this subject [10]. Of the most important signals from the 4q25 locus, only one polymorphism (rs2200733) was associated with the results of PVI and only in shortterm observation. We were unable to confirm any effect of rs10033464 in short-term or long-term observation. It seems likely that we are still far from precisely estimating the magnitude of the effect of the $4 q 25$ polymorphisms on the results of PVI in patients with AF; by the standards of genetic association studies, the number of patients in either study is very low. Therefore both studies should rather be seen as putting forward a clinical hypothesis that should be confirmed in further studies on larger cohorts or by metaanalyses. The differences between our group and that of the previously published study may offer other explanations of the dissimilarities. These differences include the following: our patients were younger with a lower frequency of lone AF (30\% vs. $83 \%$ ); we used a non-irrigated ablation catheter; we commonly isolated the vena cava superior (almost $55 \%$ of the group); and our recurrence rate after 6 months of observation was substantially higher (44\% vs. 21\%) [10]. Finally, Husser et al. accepted a $p$ value $<0.05$ as significant, without adjustment for multiple comparisons. All these factors could have caused the observed differences in results.

Our results much more resemble those published by Shoemaker et al. [14]. We also did not confirm the value of rs10033464. The nature of the relationship between rs2200733 and recurrences after pulmonary vein isolation is similar in both papers: Shoemaker et al. observed a shorter time
Table IV. Multivariate analysis of rs2200733 TT genotype and variables associated with AF recurrence within 6 months in univariate analyses. $P$-values $<0.05$ are boldfaced

\begin{tabular}{|lcc|}
\hline Variable & OR (CI) & $P$-value \\
\hline rs2200733 TT & $1.87(1.17-2.99)$ & 0.008 \\
\hline RF procedure time (s) & $1.01(0.99-1.01)$ & 0.12 \\
\hline rs6838973 & $0.88(0.66-1.17)$ & 0.39 \\
\hline $\begin{array}{l}\text { Isolation of vena cava } \\
\text { superior }\end{array}$ & $0.72(0.53-0.96)$ & 0.025 \\
\hline AF 48 h after ablation & $1.28(0.94-1.74)$ & 0.12 \\
\hline
\end{tabular}

to recurrence with the same number of recurrences, while we observed a relationship in short- but not in long-term observation. The difference is in the model of the genetic relationship - Shoemaker et al. reported a dominant model, while in our group the relationship is in a recessive model [14].

We found that the TT genotype of rs2200733 polymorphism might influence the short-term results of the first PVI. The mechanism of this relationship is unclear. There are only a few reports showing some relationships of the TT genotype with clinical variables. It is not associated with age at diagnosis, gender, family history of AF, body mass index, or AF type [15]. To the best of our knowledge, only two factors have been found to be linked with the TT genotype: prolongation of PR interval [15] and larger pulmonary veins [9]. Neither of these factors was linked with the efficacy of catheter ablation [16].

A potential link between $4 q 25$ polymorphisms and effect of treatment of AF with pulmonary vein isolation was previously described [10]. The nearest gene, PITX2 (paired-like homeodomain transcription factor 2), may play a role in the development of pulmonary myocardial sleeves [17], which are isolated during AF ablation (PVI). PITX2 is expressed in adult humans and mice predominantly in the left atrium and is downregulated in the left and right atrial tissue of patients with AF [18]. In a mouse model, Pitx $2 \mathrm{C}+/$ - hearts were more susceptible to AF during programmed stimulation (probably due to significant shortening of action potential duration) [18]. Our results suggest that only the most prominent signals from the 4 q25 locus might be important in predicting results of PVI and in short term only, whereas other polymorphisms associated with AF (1q21 and 16q22 loci) do not influence the results of PVI. The $1 q 21$ locus is at the KCNN3 gene, which encodes a member of a family of calcium-activated potassium channels [7]. The $16 q 22$ locus is located in the zinc finger homeobox 3 (ZFHX3) gene [6].

In multivariate analysis another factor significantly influenced the 6-month results of PVI: iso- 
lation of the superior vena cava. It was previously shown that isolation of the superior vena cava could improve the results of AF ablation [19]. Results of randomized trials are inconsistent, showing better results in patients with paroxysmal AF [20] or no reduction in AF recurrence [21]. Our results confirm that in some patients isolation of the VCS might be helpful.

There are several important limitations of our methodology: use of a non-irrigated catheter (now circumferential pulmonary vein isolation with an irrigated tip catheter is standard also with contact force [22], although guidelines published in 2007 did not make a firm recommendation regarding the optimal RF energy delivery and catheter [23]; results published by Cappato did not show a significant difference in AF recurrence between patients treated with irrigated and non-irrigated catheters [2]); the post-ablation monitoring was not standardized in the long term (thus potentially underestimating recurrence rates); and the difference in follow-up strategy between patients with paroxysmal and persistent AF as to the use of AAD. All these factors reduce the value of this analysis.

It should also be noted that our efficacy is rather low in short-term observation [2], but our longterm efficacy, although still low, is comparable to other published results [11, 13, 24].

In conclusion, there is a trend in the relationship between TT genotype of the rs2200733 polymorphism and increased rate of AF recurrence after PVI in short-term (6 months) follow-up. None of the tested $4 q 25,16 q 22$, and 1q21 SNPs correlated with the results of a single $A F$ ablation in long-term follow-up.

\section{Acknowledgments}

This work was supported by the Medical University of Warsaw, grants $1 \mathrm{WY} / \mathrm{N} / 10$ and $1 \mathrm{WY} / \mathrm{N} / 11$.

We thank Michal Peller for his help in statistical calculations.

\section{Conflict of interest}

The authors declare no conflict of interest.

\section{References}

1. Calkins H, Kuck KH, Cappato R, et al. 2012 HRS/EHRA/ ECAS Expert Consensus Statement on Catheter and Surgical Ablation of Atrial Fibrillation: recommendations for patient selection, procedural techniques, patient management and follow-up, definitions, endpoints, and research trial design. Europace 2012; 14: 528-606.

2. Cappato R, Calkins H, Chen SA, et al. Updated Worldwide Survey on the methods, efficacy, and safety of catheter ablation for human atrial fibrillation. Circ Arrhythm Electrophysiol 2010; 3: 32-8.
3. Ertek S, Cicero AF. Hyperthyroidism and cardiovascular complications: a narrative review on the basis of pathophysiology. Arch Med Sci 2013; 9: 944-52.

4. Gudbjartsson DF, Arnar DO, Helgadottir A, et al. Variants conferring risk of atrial fibrillation on chromosome 4q25. Nature 2007; 448: 353-7.

5. Lubitz SA, Sinner MF, Lunetta KL, et al. Independent susceptibility markers for atrial fibrillation on chromosome 4q25. Circulation 2010; 122: 976-84.

6. Gudbjartsson DF, Holm H, Gretarsdottir S, et al. A sequence variant in $\mathrm{ZFHX3}$ on 16q22 associates with atrial fibrillation and ischemic stroke. Nat Genet 2009; 41: 876-8.

7. Ellinor PT, Lunetta KL, Glazer NL, et al. Common variants in KCNN3 are associated with lone atrial fibrillation. Nat Genet 2010; 42: 240-4.

8. Kitamura K, Miura H, Miyagawa-Tomita S, et al. Mouse Pitx2 deficiency leads to anomalies of the ventral body wall, heart, extra- and periocular mesoderm and right pulmonary isomerism. Development 1999; 126: 5749-58.

9. Kiliszek M, Franaszczyk M, Kozluk E, et al. Association between variants on chromosome 4q25, 16q22 and $1 \mathrm{q} 21$ and atrial fibrillation in the Polish population. PLoS One 2011; 6: e21790.

10. Husser D, Adams V, Piorkowski C, Hindricks G, BolImann A. Chromosome 4q25 variants and atrial fibrillation recurrence after catheter ablation. J Am Coll Cardiol 2010; 55: 747-53.

11. Tzou WS, Marchlinski FE, Zado ES, et al. Long-term outcome after successful catheter ablation of atrial fibrillation. Circ Arrhythm Electrophysiol 2010; 3: 237-42.

12. Hansen SK, Gjesing AP, Rasmussen SK, et al. Largescale studies of the Hphl insulin gene variable-number-of-tandem-repeats polymorphism in relation to type 2 diabetes mellitus and insulin release. Diabetologia 2004; 47: 1079-87.

13. Weerasooriya R, Khairy P, Litalien J, et al. Catheter ablation for atrial fibrillation: are results maintained at 5 years of follow-up? J Am Coll Cardiol 2011; 57: 160-6.

14. Shoemaker BM, Muhammad R, Parvez B, et al. Common atrial fibrillation risk alleles at $4 q 25$ predict recurrence after catheter-based atrial fibrillation ablation. Heart Rhythm 2013; 10: 394-400.

15. Goodloe AH, Herron KJ, Olson TM. Uncovering an intermediate phenotype associated with rs2200733 at 4q25 in lone atrial fibrillation. Am J Cardiol 2011; 107: 1802-5.

16. den Uijl DW, Tops LF, Delgado V, et al. Effect of pulmonary vein anatomy and left atrial dimensions on outcome of circumferential radiofrequency catheter ablation for atrial fibrillation. Am J Cardiol 2011; 107: 243-9.

17. Mommersteeg MT, Brown NA, Prall OW, et al. Pit $2 \mathrm{c}$ and $\mathrm{Nk} \times 2-5$ are required for the formation and identity of the pulmonary myocardium. Circ Res 2007; 101: 902-9.

18. Kirchhof P, Kahr PC, Kaese S, et al. PITX2C is expressed in the adult left atrium, and reducing Pitx2c expression promotes atrial fibrillation inducibility and complex changes in gene expression. Circ Cardiovasc Genet 2011; 4: 123-33.

19. Arruda M, Mlcochova H, Prasad SK, et al. Electrical isolation of the superior vena cava: an adjunctive strategy to pulmonary vein antrum isolation improving the outcome of AF ablation. J Cardiovasc Electrophysiol 2007; 18: 1261-6.

20. Corrado A, Bonso A, Madalosso M, et al. Impact of systematic isolation of superior vena cava in addition to pulmonary vein antrum isolation on the outcome of paroxysmal, persistent, and permanent atrial fibrilla- 
tion ablation: results from a randomized study. J Cardiovasc Electrophysiol 2010; 21: 1-5.

21. Wang XH, Liu X, Sun YM, Shi HF, Zhou L, Gu JN. Pulmonary vein isolation combined with superior vena cava isolation for atrial fibrillation ablation: a prospective randomized study. Europace 2008; 10: 600-5.

22. Wutzler A, Huemer M, Parwani AS, Blaschke F, Haverkamp W, Boldt LH. Contact force mapping during catheter ablation for atrial fibrillation: procedural data and one-year follow-up. Arch Med Sci 2014; 10: 266-72.

23. Calkins H, Brugada J, Packer DL, et al. HRS/EHRA/ECAS Expert Consensus Statement on catheter and surgical ablation of atrial fibrillation: recommendations for personnel, policy, procedures and follow-up. Europace 2007; 9: 335-79.

24. Gaita F, Caponi D, Scaglione M, et al. Long-term clinical results of 2 different ablation strategies in patients with paroxysmal and persistent atrial fibrillation. Circ Arrhythmia Electrophysiol 2008; 1: 269-75. 